\title{
Tensile Forces in Lift Carrier Ropes Exerted by the Fluid Pressure
}

\author{
Leopold Hrabovský \\ 1 VŠB - Technical University of Ostrava, 17. listopadu 2172/15, 70800 Ostrava - Poruba, Czech Republic \\ e-mail: leopold.hrabovsky@vsb.cz
}

\begin{abstract}
This paper describes the methodology by which it is possible to compare different tensile forces that are caused by the uneven distribution of loads in carrier ropes of lifts. In order to compare the applied tensile forces in a certain number of carrier ropes, it is possible to use the device called "rope hydraulic tension compensator", when, for example, building new elevators, changing carrier ropes during renovations or servicing existing lifts. The comparison of tensile forces in lift carrier ropes by the described device is based on the method of liquid pressure diffusion in a closed vessel. The rope hydraulic tension compensator consists of a number of structural parts, the main of which are double-acting hydraulic cylinders. The number of these cylinders is equal to the number of carrier ropes of the given lift. The pressure of the hydraulic fluid, which is supplied to the inner shell of individual hydraulic cylinders, controls the length of extension of individual piston rods of the hydraulic cylinders. The position of the piston in each of the hydraulic cylinders is directly proportional to the length of the extended piston rod, which corresponds to compression of the cylindrical coiled spring, above which the respective hydraulic cylinder is located. Different compressions of individual cylindrical springs exert different magnitude of tensile forces in suspension eye-bolts, into whose longitudinal axes the total load size is distributed unevenly. The paper also describes the description and hydraulic circuit diagram of a hydraulic power unit, which is used to control the piston positions of two-way hydraulic cylinders.
\end{abstract}

Keywords: tensile force, lift, carrier rope, fluid pressure.

\section{INTRODUCTION}

Several papers have been devoted to the issue, as well as to possible solutions of how to equalize different values of tensile forces in lift carrier ropes $[1,2,3]$. These papers described structural solutions and principles of device, the so-called mechanical rope tension compensators which allow to detect instantaneous values of tensile forces in individual cross-sections of carrier ropes of traction rope lifts and to adjust any possibly different tensile forces to the same value. These devices use strain gauge load cells [3] or foil strain gauges $[1,2]$.

Traction rope electric lifts use friction between carrier ropes and grooves of the lift machine traction disk to exert a vertical movement of the lift cage.
Carrier ropes of traction lifts, see Fig. 1, with rope transmission 1:1, are attached to the lift cage and counterweight by means of spring hinges. By using a 2: 1 rope transmission both ends of carrier ropes are fixed by spring hinges to the brackets in the elevator shaft.

The load capacity of the lift, i.e. the maximum weight of the load, assigns the given number of ropes to the traction lift, however, two carrier ropes are the minimum. The tensile force, exerted by the weight of the cage and the maximum weights of the load, affecting the carrier ropes of traction lifts must be evenly distributed over all cross-sections of the ropes. The tensile force can be evenly distributed across all cross-sections of the ropes using one of the variants of rope mechanical tension compensators $[1,2,3]$, rope sensors [4] or rope hydraulic tension compensator [5]. 

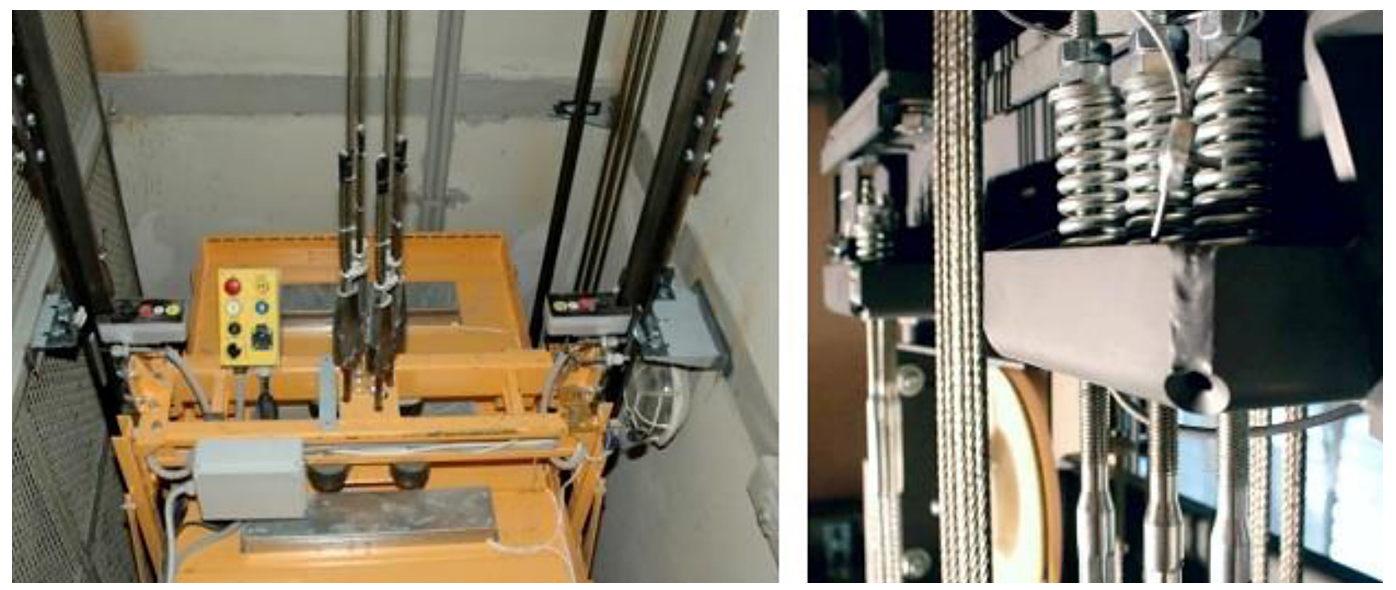

Fig. 1. Spring hinge of lift carrier ropes

The rope hydraulic tension compensator, see Fig. 2, allows to compensate for different tensile forces operating in the lift carrier ropes, based on the principle of Pascal's law, which generally defines that the pressure exerted anywhere in closed incompressible fluid spreads uniformly in all directions throughout the volume of the liquid.

The spring hinge of lift carrier ropes, see Fig. 3, consists of a suspension eye-bolt $\underline{\mathrm{A}}$, a cylindrical compression coiled spring $\underline{B}$, two plates $\underline{C}, \underline{D}$, a washer $\underline{G}$ and a hexagonal nut $\underline{F}$ and a lock nut $\mathrm{E}$. The end of each carrier rope is threaded through the eye of the suspension bolt $\underline{A}$ and by means of rope clamps or pressed sleeves an eye is formed on the carrier rope. The threaded parts of suspension bolts with eyes $\underline{A}$ are threaded

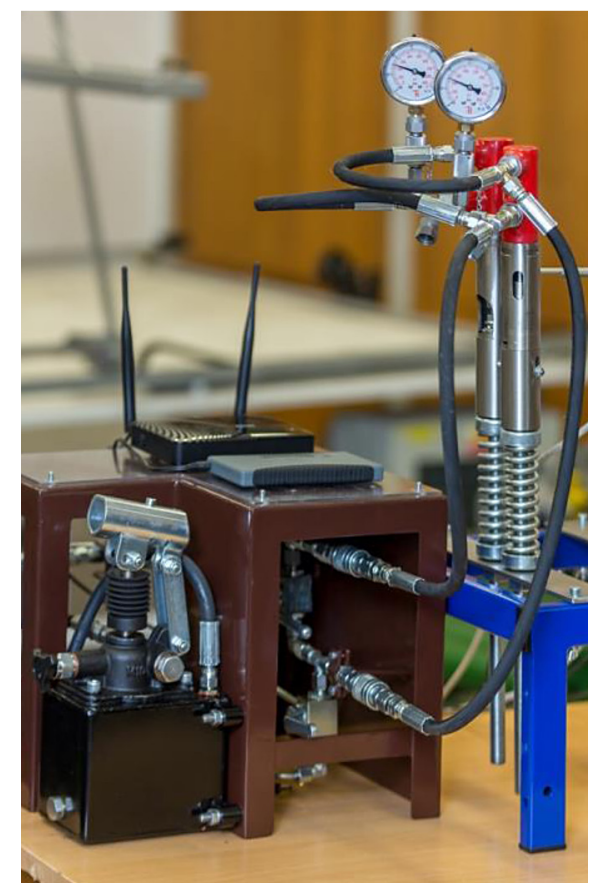

Fig. 2. Rope hydraulic tension compensator through the holes in the bracket, which is fixed to the wall of the lift well.

In the space above the upper surface of the bracket, on the shank of the suspension bolt with eye $\underline{A}$, a plate $\underline{C}$ is threaded, the inner hole of which serves for fitting of the cut front part of the closing thread of the cylindrical spring $\underline{B}$. The plate $\underline{D}$ is threaded onto the opposite end of the cylindrical spring $\underline{B}$. To prevent the bolt shank from sliding out of the bracket opening, the washer $\underline{\mathrm{G}}$ is threaded onto the bolt shank and the hexagonal nuts $\underline{F}$ are screwed onto the threaded part of the bolt, which is secured against loosening by the lock nut $\underline{E}$. Cylindrical compression springs $\underline{B}$ of all hinge bolts $\underline{\mathrm{A}}$ have the same stiffness (spring stiffness, defined by the relationship $\mathrm{k}=\mathrm{F} / \Delta \mathrm{l}[-]$, indicating the rate of change in its length $\Delta \mathrm{l}[\mathrm{m}]$ with respect to the acting external force $\mathrm{F}[\mathrm{N}]$ at elastic deformation.).

Different magnitude of acting tensile forces $F_{i}$ $[\mathrm{N}]$ in the carrier ropes (the total number of ropes $\mathrm{i}$ $=1, \ldots, n)$ causes, with identical stiffness to [-] cylindrical spiral springs, different values of spring deformation $\Delta \mathrm{l}_{\mathrm{i}}[\mathrm{m}]$, see the relationship (1).

$$
\begin{aligned}
& \mathrm{F}_{1} \neq \mathrm{F}_{\mathrm{i}} \neq \mathrm{F}_{\mathrm{n}} \Rightarrow \\
& \Rightarrow \mathrm{F}_{1}=\mathrm{k} . \Delta \mathrm{l}_{1} \neq \mathrm{F}_{\mathrm{i}}=\mathrm{k} \cdot \Delta \mathrm{l}_{\mathrm{i}} \neq \mathrm{F}_{\mathrm{n}}=\mathrm{k} \cdot \Delta \mathrm{l}_{\mathrm{n}} \Rightarrow \\
& \Rightarrow \Delta \mathrm{l}_{1} \neq \Delta \mathrm{l}_{\mathrm{i}} \neq \Delta \mathrm{l}_{\mathrm{n}}[\mathrm{m}]
\end{aligned}
$$

With hydraulic cylinders (with identical construction dimensions and piston diameters $\mathrm{d}[\mathrm{m}]$ ) mounted on the end part of suspension bolts, the pressure $\mathrm{p}[\mathrm{Pa}$ ] of hydraulic fluid (distributed by the hydraulic piping into the space under the pistons of all hydraulic cylinders) makes it possible to have primarily different values of acting tensile forces $F_{i}[N]$ in the carrier ropes modified to the same value $\mathrm{F}[\mathrm{N}]$. 

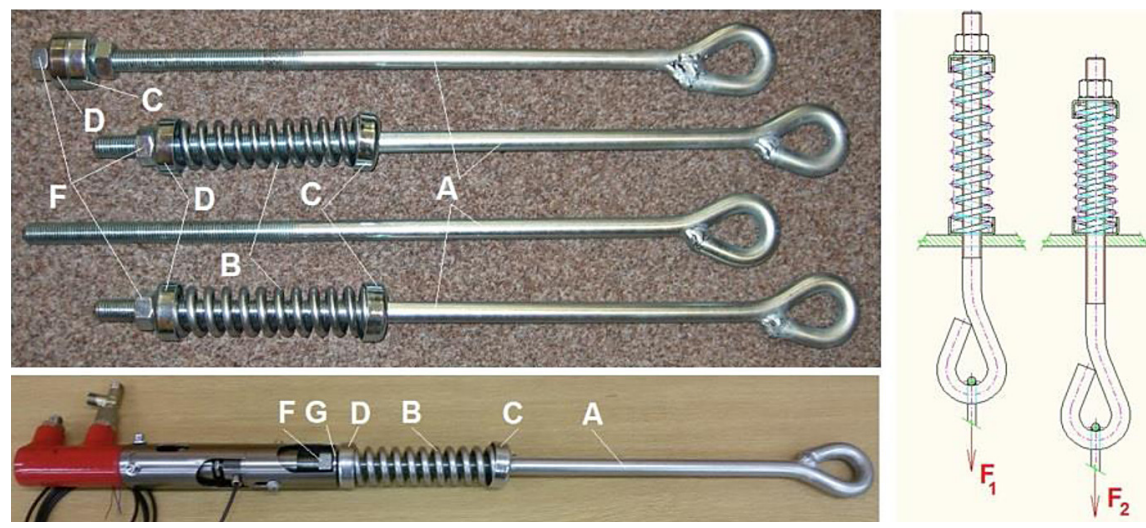

Fig. 3. Spring hinge of lift carrier ropes

\section{PRINCIPLE OF HYDRAULIC TENSION COMPENSATOR IN CARRIER ROPES}

After the assembly of all spring hinges to the bracket in the lift well (or to the lift cage frame) (Fig. 1), and after installation of the carrier ropes, the lift cage and the counterweight, the compression coil springs B (Fig. 3) of the spring hinges are deformed differently due to the uneven distribution of applied load into the selected number of carrier ropes, which can be described by relationship (1).

Each fully extended piston rod (exerted by the pressure of the hydraulic fluid supplied above the pistons of hydraulic cylinders, see Fig. 6a) from the two-way hydraulic cylinders (the number of cylinders used is identical to the number of carrier ropes) is mechanically connected to the end portion of the thread of the suspension bolt. Since different tensile forces (acting in the individual carrier ropes) are compressed differently by the springs, the ends of hydraulic cylinders have different distance from the upper surface of the bracket.

Two cylindrical bodies ( $2 \mathrm{a}, 2 \mathrm{~b}$, see Fig. 4 ) of the same overall length are always inserted between the front part of each hydraulic cylinder and the upper surface of the plate $(\mathrm{D}$, see Fig. $3)$. The maximum length of the two cylindrical bodies (2a, 2b, see Fig. 4) is selected according to the design of the hydraulic cylinders used, namely according to the maximum possible length of extension of piston rods from the shell of hydraulic cylinders.

By using the cylindrical bodies (2a, 2b, see Fig. 4), which precisely define the distance between the front part of a particular hydraulic cylinder and the upper surface of the plate ( $\underline{D}$, see Fig. 3) of a particular suspension screw, the front parts of the hydraulic cylinders are still different from the upper surface of the bracket.

To control the extension of the piston rod from the shell of the two-way hydraulic cylinder of the hydraulic tension compensator, a hydraulic power unit has been created, (Fig. 5). The circuit diagram of the hydraulic circuit of the hydraulic power unit is shown in Figure 6. The hydraulic power unit consists of manual hydraulic pump $\underline{1}$, one-way valve $\underline{2}$, two twoway valves $\underline{3}$ and $\underline{4}$, hydraulic quick-couplings $\underline{5}$ and $\underline{6}$, which are connected to each other by a hydraulic pipeline.

To compensate for the different tensile force values Fi $[\mathrm{N}]$ in individual carrier ropes, the hydraulic fluid is pumped after installation of all two-way hydraulic cylinders (Fig. 2) on the suspension bolt shanks using the lever of manual hydraulic pump 1 through the pipeline and through

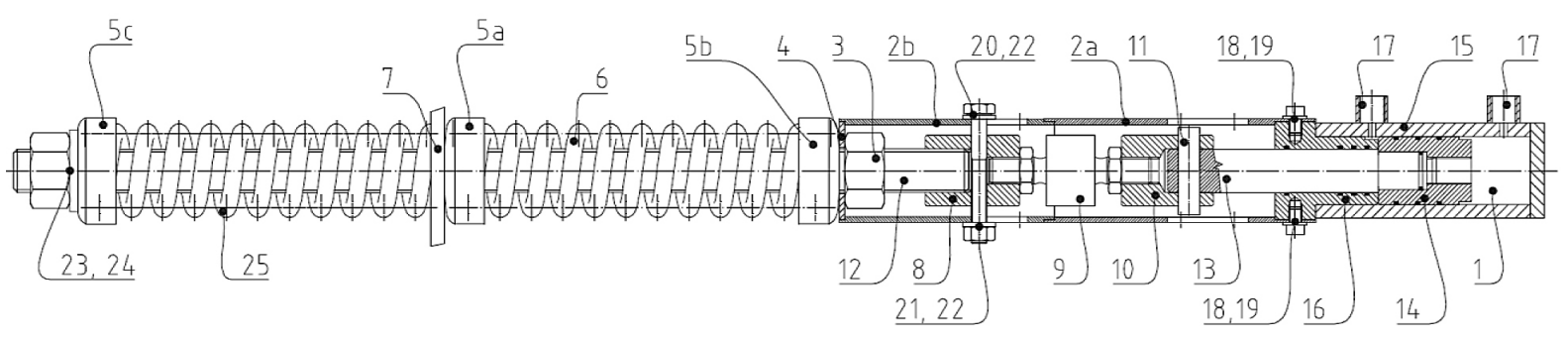

Fig. 4. Draft design of the mobile hydraulic tension compensator 

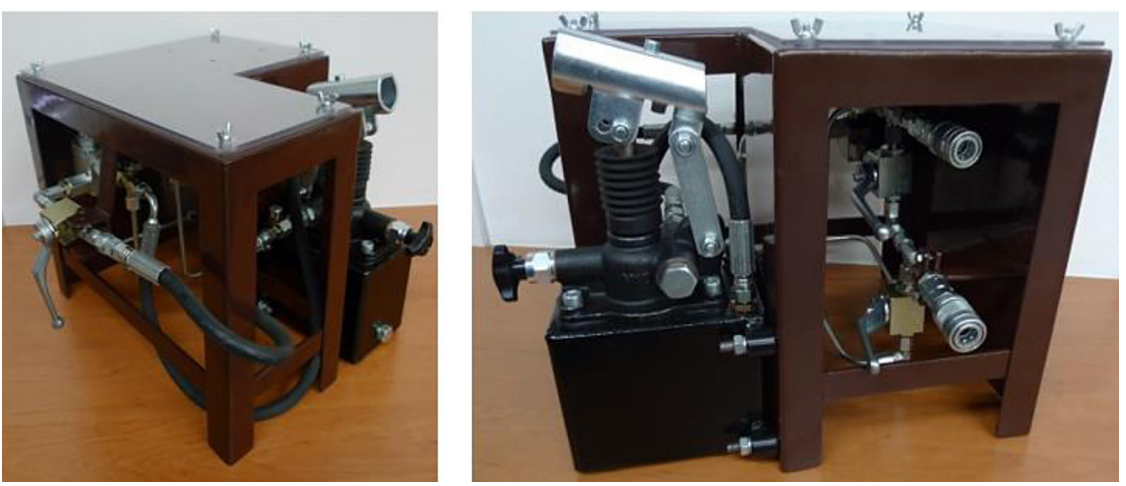

Fig. 5. Hydraulic power unit controlling piston rods of double acting hydraulic cylinders of rope hydraulic tension compensator

the open one-way valve $\underline{2}$ and two-way valve $\underline{3}$ that is adjusted to suitable position (see Fig. 6b) to the quick-coupling 5 . Hydraulic hoses which bring the same fluid pressure under the pistons of hydraulic cylinders $\underline{7 \mathrm{a}}$ and $\underline{7 \mathrm{~b}}$ are connected to the quick-coupling $\underline{5}$. Other hydraulic hoses also interconnect the spaces above the pistons of hydraulic cylinders $\underline{7 \mathrm{a}}$ and $\underline{7 \mathrm{~b}}$.

Due to the increasing pressure $\mathrm{p}[\mathrm{Pa}]$ of the hydraulic fluid under the pistons of all hydraulic cylinders, the spring with the smallest load is compressed as first (its length $1 i=1$ max (i) $[\mathrm{m}]$, and if the same compression of this spring is achieved with the spring with the biggest load, all springs are subsequently compressed by the same size. Then, due to the applied hydraulic fluid pressure $\mathrm{p} 2[\mathrm{~Pa}]$ under the pistons of the hydraulic cylinders $\underline{7 \mathrm{a}}$ and $\underline{7 \mathrm{~b}}$ the relationship (2) applies which states that the initially different tensile forces in the carrier ropes $\mathrm{F}$ (i) [N] already reach the same magnitude $\mathrm{F}[\mathrm{N}]$.

$$
\begin{aligned}
& \mathrm{p}_{2}=\frac{\mathrm{F}_{\mathrm{i}}+\Delta \mathrm{F}_{\mathrm{i}}}{\mathrm{S}}=\frac{\mathrm{F}}{\mathrm{S}} \Rightarrow \\
& \Rightarrow \mathrm{F}_{1}+\Delta \mathrm{F}_{1}=\mathrm{F}_{\mathrm{i}}+\Delta \mathrm{F}_{\mathrm{i}}+\ldots+\mathrm{F}_{\mathrm{n}}+\Delta \mathrm{F}_{\mathrm{n}}=\mathrm{F}[\mathrm{N}]
\end{aligned}
$$

Since the lower surfaces of cylindrical bodies $\underline{2 b}$ (see Fig. 4) lean against the upper surfaces of plates $\underline{5 \mathrm{~b}}$ and also because the cylindrical bodies $\underline{2 a}$ are attached to the shells of hydraulic cylinders, at the time when hydraulic fluid at a certain (gradually increasing) pressure is supplied by the hydraulic power unit under the pistons of hydraulic cylinders, the piston rods are gradually inserted into the shells of the hydraulic cylinders. The piston rods of individual hydraulic cylinders are mechanically attached to the ends of the shanks of respective suspension bolts, so the length of insertion of the piston rod into the shell of individual hydraulic cylinders is not (with the same instantaneous pressure $\mathrm{p}[\mathrm{Pa}]$ of hydraulic fluid in the space below the pistons) of the same size. Each of the piston rods is inserted into the shell of the hydraulic cylinder by a different length $\Delta \mathrm{L}_{\mathrm{i}}$ [m], which is directly proportional to the tensile load (and the instantaneous pressure of the hydraulic fluid acting under the piston of the respective hydraulic cylinder) acting in the axis of the suspension bolt to which the respective hydraulic cylinder is connected. At the same magnitude of gradually increasing hydraulic fluid pressure (applied below the pistons of all hydraulic cylinders),

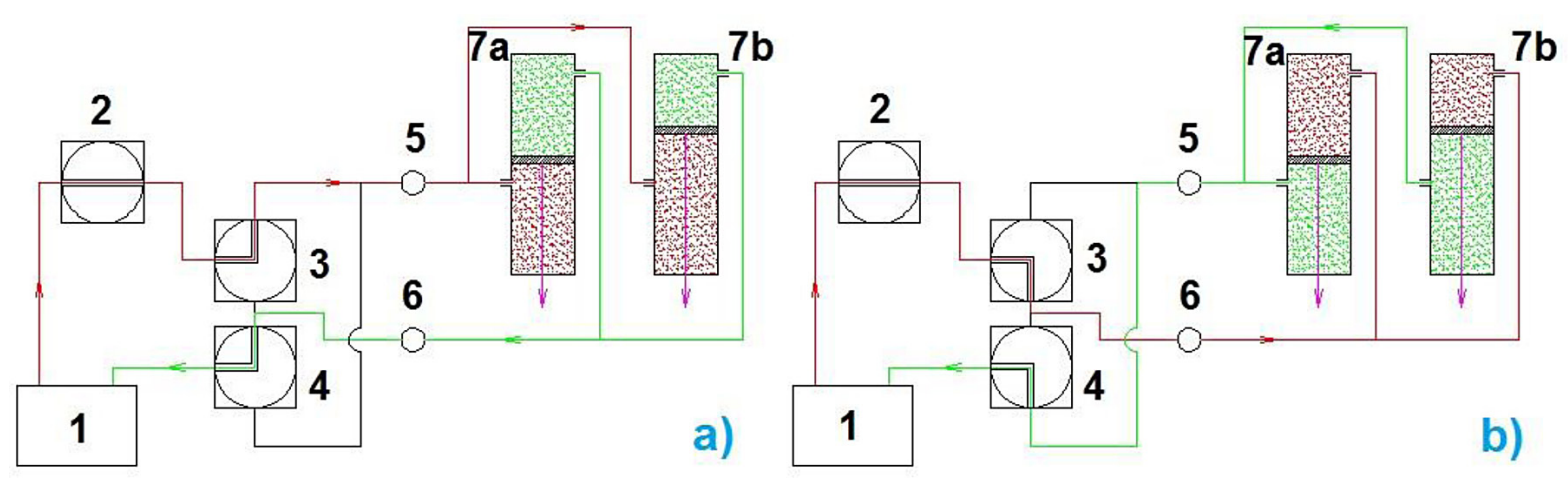

Fig. 6. Diagram of the hydraulic circuit of the hydraulic power unit 
the piston rod is initially inserted into the shell of the hydraulic cylinder attached to the suspension bolt which is affected by the smallest tensile force (because the carrier rope has the smallest load). Piston rods retracted (different length values $\Delta \mathrm{L}_{\mathrm{i}}$ $[\mathrm{m}]$ ) into the shells of hydraulic cylinders produce different tensile forces $\mathrm{F}_{\mathrm{i}}[\mathrm{N}]$, which affect the respective suspension bolts. Due to the different tensile forces $\mathrm{F}_{\mathrm{i}}[\mathrm{N}]$, the end parts of shanks of the individual suspension bolts begin (by increasing the hydraulic fluid pressure below the pistons of hydraulic cylinders) to move upwards (relative to the top surface of the bracket) by a different size of $\Delta \mathrm{L}_{\mathrm{i}}[\mathrm{m}]$, which is proportional to the amount of load affecting the carrier rope.

The shells of hydraulic cylinders are fastened to cylindrical bodies 2 a by means of screws which have identical and constant design lengths (see Fig. 4). Cylindrical bodies 2a are interconnected with cylindrical bodies $\underline{2 b}$, which are also designed to have the same length. The lower surfaces of cylindrical bodies $\underline{2 b}$ lean on the upper surfaces of plates $\underline{5 b}$, and that is why, when the piston rods (with different length $\Delta \mathrm{L}_{\mathrm{i}}[\mathrm{m}]$ ) are inserted into the shells of hydraulic cylinders, the tensile forces $\mathrm{F}_{i}[\mathrm{~N}]$ are transferred to cylindrical springs, which are deformed differently by these different forces $\mathrm{F}_{\mathrm{i}}[\mathrm{N}]$. In simplified terms, it can be defined that the known value of the insertion length $\Delta \mathrm{L}_{\mathrm{i}}[\mathrm{m}]$ of the piston rod into the shell of a particular hydraulic cylinder, at a specific pressure $\mathrm{p}_{\mathrm{i}}[\mathrm{Pa}]$ below the piston, causes the compression of the spring $\Delta \mathrm{l}_{\mathrm{i}}[\mathrm{m}]$.

If the same design of all ( $\mathrm{i}$-th number) compression cylindrical springs is selected for spring hinges of carrier cables of the given lift, the length of all springs without load is $1_{0}[\mathrm{~m}]$.

At the instantaneous liquid pressure $p_{i}[\mathrm{~Pa}]$ below the piston of the $\mathrm{i}$-th hydraulic cylinder, the $i$-th spring length can be expressed as $\Delta \mathrm{L}_{\mathrm{i}}[\mathrm{m}]$ and the instantaneous compression of the $\Delta \mathrm{L}_{\mathrm{i}}[\mathrm{m}]$ of this spring by the relationship (3).

$$
\Delta \mathrm{L}_{\mathrm{i}}=1_{0}-1_{\mathrm{i}}[\mathrm{m}]
$$

If the length of the spring with the biggest load is labelled as $1_{\min (i)}[\mathrm{m}]$ then at some point when the pressure of the liquid under the piston just reaches the value of $\mathrm{p}_{2}[\mathrm{~N}]$ (the pressure value $p_{i}[\mathrm{~Pa}]$ corresponds to the length of the loaded spring to the length $1_{\min (\mathrm{i})}[\mathrm{m}]$ of the $\mathrm{i}$-th spring that had the biggest load at the initial moment), the length of all springs can be expressed as $1_{\min (\mathrm{i})}[\mathrm{m}]$ and the instantaneous compression of all springs $\Delta \mathrm{L}_{\max (\mathrm{i})}[\mathrm{m}]$ by the relationship (4).

$$
\Delta \mathrm{L}_{\max (\mathrm{i})}=1_{0}-1_{\min (\mathrm{i})}[\mathrm{m}]
$$

When the pressure $p_{2}[N]$ is reached, all springs reach the same length $1_{(\mathrm{i})}=1_{\min (\mathrm{i}}[\mathrm{m}]$, which equals to the length of the spring with the biggest load $1_{\min (\mathrm{i})}[\mathrm{m}]$ at the fluid pressure $\mathrm{p}_{\mathrm{i}}<\mathrm{p}_{2}[\mathrm{~Pa}]$ of the spring that had the biggest load as first, it is possible to observe from the relationship (4) that if the pressure $p_{2}[N]$ is just attained then all springs take the length $1_{(\mathrm{i})}=1_{\min (\mathrm{i})}[\mathrm{N}]$.

With further pressure increase $\mathrm{p}_{3}>\mathrm{p}_{2}[\mathrm{~Pa}]$, the same compression size $\Delta \mathrm{L}[\mathrm{m}]$ of all spiral compression springs occurs and all springs have the same length $1[\mathrm{~m}]$.

If the pressure in the liquid $\mathrm{p}_{2}[\mathrm{~Pa}]$ is reached below the pistons of hydraulic cylinders, then the compressive force $\mathrm{F}[\mathrm{N}]$ (proportional to the highest value of all the loads acting on all the springs before installation of tension compensator) acts on the first most compressed spring $\underline{6}$ (Fig. 4). Also, for all other springs $\underline{6}$ of all suspension bolts, this pressure force $\mathrm{F}[\mathrm{N}]$ acts when the pressure in the liquid $\mathrm{p}_{2}[\mathrm{~Pa}]$ is reached below the pistons of the hydraulic cylinders.

By increasing the applied pressure under the pistons of the hydraulic cylinders above the pressure $\mathrm{p}_{2}[\mathrm{~Pa}]$, all the springs $\underline{6}$ (Fig. 4 ) are compressed by the compressive force transmitted by the cylindrical bodies $\underline{2 a}, \underline{2 b}$ from the hydraulic cylinders $\underline{1}$. The shanks of the suspension bolts $\underline{12}$ are lifted by the rods 13 of the hydraulic cylinder $\underline{1}$ and the nuts $\underline{3}$ are removed from the plates $\underline{5 b}$. By tightening all nuts $\underline{3}$ with the same moment of force (i.e. all nuts $\underline{3}$ are tightened so that all spring lengths are the same), the required uniform load distribution is achieved across all cross-sections of the carrier ropes.

At the time of supply of the pressurized hydraulic fluid (pressure increase $\mathrm{p}[\mathrm{Pa}]$ ) under the pistons of hydraulic cylinders ( $\underline{7 a}, \underline{7 b}$, see Fig. $6 \mathrm{~b})$, the liquid is drawn from the spaces above the pistons of hydraulic cylinders $\underline{7 \mathrm{a}}, \underline{7 \mathrm{~b}}$ by the hydraulic hose to the quick-coupling $\underline{6}$. The hydraulic fluid is routed through the hydraulic power unit line (Fig. 5) via a two-way valve $\underline{4}$ (adjusted to a suitable position, see Fig. $6 \mathrm{~b}$ ) to the hydraulic pump tank $\underline{1}$.

If a uniform load distribution to all crosssections of carrier ropes is achieved as described above, all components of the rope hydraulic 
tension compensator must be removed from the screw hinge of carrier ropes. When disassembling it is necessary to extend all piston rods from hydraulic cylinders to the maximum possible position. This is ensured by the hydraulic power unit. The lever of the manual hydraulic pump 1 pumps the hydraulic fluid through a line through the open one-way valve $\underline{2}$ and the two-way valve $\underline{3}$ (adjusted to a suitable position, see Fig. 6a) to the quick-coupling $\underline{6}$. A hydraulic hose is connected to the quick-coupling $\underline{6}$ which supplies the liquid above the pistons of hydraulic cylinders $\underline{7 a}$, $\underline{7 b}$. Another hydraulic hose connects the spaces under the pistons of hydraulic cylinders $7 \mathrm{a}$ and $\underline{7 b}$. The fluid from under the pistons of hydraulic cylinders $\underline{7 \mathrm{a}}$ and $\underline{7 \mathrm{~b}}$ is discharged through the hydraulic hose to the quick-coupling $\underline{5}$. The hydraulic fluid is routed via a pipeline in the hydraulic power unit via a two-way valve $\underline{4}$ (adjusted to a suitable position, see Fig. 6a) into the hydraulic pump tank $\underline{1}$.

With further pressure increase $\mathrm{p}_{3}>\mathrm{p}_{2}[\mathrm{~Pa}]$, the same compression size $\Delta \mathrm{L}[\mathrm{m}]$ of all spiral compression springs occurs and all springs have the same length $1[\mathrm{~m}]$.

If the pressure in the liquid $\mathrm{p}_{2}[\mathrm{~Pa}]$ is reached below the pistons of hydraulic cylinders, then the compressive force $\mathrm{F}[\mathrm{N}]$ (proportional to the highest value of all the loads acting on all the springs before installation of tension compensator) acts on the first most compressed spring $\underline{6}$ (Fig. 4). Also, for all other springs $\underline{6}$ of all suspension bolts, this pressure force $\mathrm{F}[\mathrm{N}]$ acts when the pressure in the liquid $\mathrm{p}_{2}[\mathrm{~Pa}]$ is reached below the pistons of the hydraulic cylinders.

By increasing the applied pressure under the pistons of the hydraulic cylinders above the pressure $\mathrm{p}_{2}[\mathrm{~Pa}]$, all the springs $\underline{6}$ (see Fig. 4 ) are compressed by the compressive force transmitted by the cylindrical bodies $\underline{2 a}, \underline{2 b}$ from the hydraulic cylinders $\underline{1}$. The shanks of the suspension bolts $\underline{12}$ are lifted by the rods 13 of the hydraulic cylinder $\underline{1}$ and the nuts $\underline{3}$ are removed from the plates $\underline{5 b}$. By tightening all nuts $\underline{3}$ with the same moment of force (i.e. all nuts $\underline{3}$ are tightened so that all spring lengths are the same), the required uniform load distribution is achieved across all cross-sections of the carrier ropes.

At the time of supply of the pressurized hydraulic fluid (pressure increase $\mathrm{p}[\mathrm{Pa}]$ ) under the pistons of hydraulic cylinders $(\underline{7 \mathrm{a}}, \underline{7 \mathrm{~b}}$, see Fig. $6 \mathrm{~b})$, the liquid is drawn from the spaces above the pistons of hydraulic cylinders $\underline{7 \mathrm{a}}, \underline{7 \mathrm{~b}}$ by the hydraulic hose to the quick-coupling $\underline{6}$. The hydraulic fluid is routed through the hydraulic power unit line (Fig. 5) via a two-way valve $\underline{4}$ (adjusted to a suitable position, see Fig. 6b) to the hydraulic pump tank $\underline{1}$.

\section{CONCLUSION}

The possible way of how to achieve uniform load distribution into two or more carrier ropes in the traction lift using a rope hydraulic tension compensator is given in the text of Chapter 2 of this paper. This device is portable and is mounted on suspension bolts only when it is necessary to set the same tensile forces in carrier ropes. After carrying out its activities, it is possible to remove the device from the suspension bolts and move it to another traction rope lift.

In contrast to the known principle of the hydraulic compensator [5], the described device can be provided with strain gauge load cells that can detect instantaneous tensile forces in carrier cables, record them and use them for certificate processing purposes.

The described rope hydraulic tension compensator can fully fulfil the function for which it has been designed without the use of strain gauge load cells. If the compensator is not equipped with a strain gauge, then the piston rod of the hydraulic cylinder $\underline{13}$ (Fig. 4 and Fig. 5) is connected to the cylindrical body 10 mechanically. The threaded end portion of the shank of bolt 12 is screwed onto the internal thread of the body $\underline{8}$. By means of a threaded rod (which replaces the strain gauge load cell) of the required length, the bodies $\underline{8}$ and $\underline{10}$ are interconnected so that the hydraulic compensator performs as described above.

Double-acting hydraulic cylinders which equalize the initially different tensile forces in the ropes to the same values are connected via hydraulic pressure hoses to the hydraulic power unit. With the hydraulic power unit, the hydraulic fluid is pumped from the tank through the manual hydraulic pump via suitably open or closed hydraulic valves to the spaces below or above the hydraulic cylinder pistons. In the "under / above the piston" space of cylinders, where the hydraulic fluid pressure is supplied by the hand pump, the piston moves in the cylinder and the hydraulic fluid from the "below / above the piston" space moves to drain the hydraulic fluid back into the tank. 


\section{Acknowledgements}

This work has been supported by The Ministry of Education, Youth and Sports of the Czech Republic from the Specific Research Project SP2019/101.

\section{REFERENCES}

1. Hrabovský, L.: Apparatus Producing an Even Distribution of Strain into Carries. 022097World Multidisciplinary Civil Engineering-ArchitectureUrban Planning Symposium'2017. Vol. 245, 2017, 1-6, DOI: 10.1088/1757-899X/245/2/022097.

2. Hrabovský, L., Michalik, P.: A tension equalizes in lidt carrying ropes. Advances in Science and Technology Research Journal, 11(4), 2017, 326-332, DOI: $10.12913 / 22998624 / 80936$.

3. Hrabovský, L., Maslarić M.: Device designed for detection and setting the required tensile force in ropes. Advances in Science and Technology Research Journal, 12(1), 2018, 200-206, DOI: $10.12913 / 22998624 / 86614$.

4. http://www.liftecgroup.com/lana.html

5. https://www.brugglifting.com/elevator/images/ downloads/manu/RLE_Manual.pdf

6. Hrabovský, L., Mantič, M., Voštová, V.: Adhesion Coefficient on the Limit of Slippage at Star-Up of the Manual Crane Trolley. Advances in Science and Technology Research Journal, 13(2), 2019, 92-99, DOI: 10.12913/22998624/106244. 